\title{
Pre-metastatic Niche Formation in Different Organs Induced by Tumor Extracellular Vesicles
}

\author{
Qi Dong 1,2,3, Xue Liu'1,2, Ke Cheng ${ }^{3}$, Jiahao Sheng ${ }^{3}$, Jing Kong ${ }^{3}$ and Tingjiao Liu',2* \\ 1 Department of Basic Science of Stomatology, Shanghai Stomatological Hospital, Fudan University, Shanghai, China, \\ ${ }^{2}$ Shanghai Key Laboratory of Craniomaxillofacial Development and Diseases, Fudan University, Shanghai, China, \\ ${ }^{3}$ Department of Oral Pathology, School of Stomatology, Dalian Medical University, Dalian, China
}

OPEN ACCESS

Edited by:

Dongmei Zhang,

Jinan University, China

Reviewed by:

Laurent Counillon,

University of Nice Sophia Antipolis,

France

Xiuping Chen,

University of Macau, China

*Correspondence:

Tingjiao Liu

tingjiao_liu@fudan.edu.cn

Specialty section:

This article was submitted to

Cellular Biochemistry,

a section of the journal

Frontiers in Cell and Developmental

Biology

Received: 30 June 2021 Accepted: 01 September 2021

Published: 20 September 2021

Citation:

Dong Q, Liu X, Cheng K, Sheng J,

Kong $J$ and LiU T (2021)

Pre-metastatic Niche Formation in Different Organs Induced by Tumor

Extracellular Vesicles.

Front. Cell Dev. Biol. 9:733627.

doi: 10.3389/fcell.2021.733627
Primary tumors selectively modify the microenvironment of distant organs such as the lung, liver, brain, bone marrow, and lymph nodes to facilitate metastasis. This supportive metastatic microenvironment in distant organs was termed the pre-metastatic niche (PMN) that is characterized by increased vascular permeability, extracellular matrix remodeling, bone marrow-derived cells recruitment, angiogenesis, and immunosuppression. Extracellular vesicles (EVs) are a group of cell-derived membranous structures that carry various functional molecules. EVs play a critical role in PMN formation by delivering their cargos to recipient cells in target organs. We provide an overview of the characteristics of the PMN in different organs promoted by cancer EVs and the underlying mechanisms in this review.

Keywords: pre-metastatic niche (PMN), extracellular vesicles (EVs), vascular permeability, extracellular matrix (ECM), bone marrow-derived cells (BMDCs), immunosuppression

\section{INTRODUCTION}

Primary tumors selectively modify the microenvironment of distant organs before metastasis (Hiratsuka et al., 2002; Kaplan et al., 2005). This supportive metastatic microenvironment in distant organs was first termed the pre-metastatic niche (PMN) by Kaplan et al. (2005). In the last decade, PMN induced by various cancers has been identified in the lung (Hiratsuka et al., 2002; Hoshino et al., 2015; Liu et al., 2016; Tyagi et al., 2021), liver (Hoshino et al., 2015; Zhang and Wang, 2015; Houg and Bijlsma, 2018; Sun et al., 2021), brain (You et al., 2020), bone (Kaplan et al., 2007; $\mathrm{Xu}$ et al., 2017), and other organs. The PMN is characterized by increased vascular permeability (Gupta et al., 2007; Huang et al., 2009; Zhou et al., 2014), a modified extracellular matrix (ECM) (Aguado et al., 2016; Kim et al., 2019; Mohan et al., 2020), recruited bone marrow-derived cells (BMDCs) (Kitamura et al., 2015; Wang et al., 2019), and immunosuppression (Chen et al., 2011; Tacke et al., 2012; Giles et al., 2016) in the future metastatic organs.

Extracellular vesicles (EVs) are a heterogeneous group of nano-sized membranous structures that are released by almost all cells into extracellular spaces and have many different physiological and pathophysiological functions (Raposo and Stoorvogel, 2013; Colombo et al., 2014). Different EV types, including exosomes, microvesicles, apoptotic bodies, oncosomes, and megasomes have been characterized on the basis of their biogenesis pathways and sizes. Among them, exosomes and microvesicles are the most intensively studied types. Exosomes have an endocytic origin and form by the fusion between multivesicular bodies and the plasma membrane, whereas microvesicles are generated by plasma membrane shedding (van Niel et al., 2018). However, it is difficult 
to classify EVs according to their biogenic origin once they are secreted into the extracellular space. Therefore, sizebased or density-based nomenclature is recommended by the International Society of Extracellular Vesicles (Thery et al., 2018). EVs contain various functional molecules (proteins, mRNAs, miRNAs, long non-coding RNAs, and double stranded DNA, etc.) that can be trafficked between cells as a means of intercellular communication at both paracrine and systemic levels (Valadi et al., 2007; Balaj et al., 2011; Choi et al., 2013; Thakur et al., 2014). EV cargos are protected by the membrane during the delivery process, which is critical for the communication between primary tumors and distant organs. EVs can be internalized by recipient cells via different mechanisms, including phagocytosis, macropinocytosis, endocytosis, and direct membrane fusion (van Niel et al., 2018). In addition, ligands on EV membrane can interact with receptors on the recipient cell surface and elicit biological responses directly.

In this mini-review, we provide an overview of the characteristics of the PMN in different organs promoted by cancer EVs. The terms, exosomes, microvesicles, or EVs were used to describe their roles in PMN formation to ensure consistency with the original articles.

\section{CHARACTERISTICS OF THE PRE-METASTATIC NICHE}

The characteristics of the PMN formed in various organs by EVs are summarized in Figure $\mathbf{1}$ and Table 1. The lung is the most commonly involved organ, followed by the liver, bone, brain, and lymph nodes (LNs). As shown in Figure 1, EVs produced by tumor and stromal cells enter the circulation and arrive at distant organs, where they trigger a sequence of local changes including increased vascular permeability, ECM remodeling, BMDC recruitment, angiogenesis, and immunosuppression.

\section{Increased Vascular Permeability}

Increased vascular permeability is an early event in PMN formation (Huang et al., 2009; Araldi et al., 2012). Vascular destabilization in the PMN promotes the extravasation of tumor cells and facilitates metastasis. Both EV-associated miRNAs and proteins contribute to vascular destabilization by destroying adhesion molecules between endothelial cells. Exosomal miR-25-3p derived from human colorectal cancer cells promotes vascular permeability in mouse models by regulating the expression of the tight junction proteins zonula occludens- 1

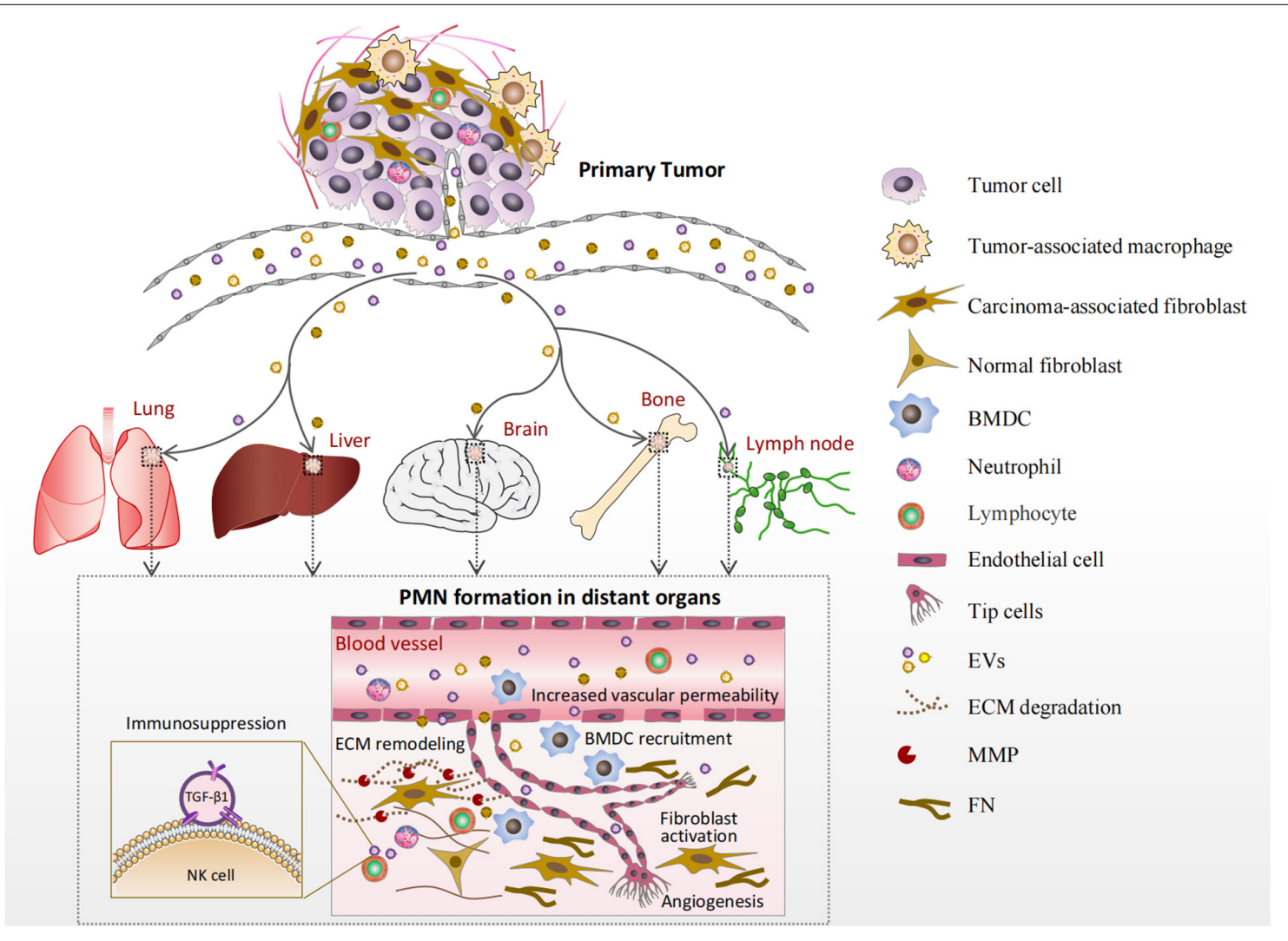

FIGURE 1 | Formation of the pre-metastatic niche (PMN) in various organs. Extracellular vesicles (EVs) produced by tumor and stromal cells enter the circulation and arrive at distant organs such as the lung, liver, brain, bone marrow, and lymph nodes (LNs). Increased vascular permeability is an early event in PMN formation. Then, EVs cause extracellular matrix (ECM) remodeling principally by activating resident normal fibroblasts. The activated fibroblasts deposit new ECM components, such as FN, and produce matrix metalloproteinases (MMPs). Bone marrow-derived cells (BMDCs) are recruited into the target organs by EVs and involved in angiogenesis and/or immune responses. Furthermore, EVs can deliver their cargos to endothelial cells or immunocytes directly in the PMN to promote angiogenesis or create an immunosuppressive microenvironment. 
TABLE 1 | Pre-metastatic niche (PMN) formation promoted by extracellular vesicles (EVs).

\begin{tabular}{|c|c|c|c|c|}
\hline Organs & Tumor & PMN-promoting EVs & PMN characteristics & References \\
\hline Lung & Breast cancer & Exosomes from EO771 cells & $\begin{array}{l}\text { BMDC recruitment and } \\
\text { immunosuppression }\end{array}$ & Wen et al., 2016 \\
\hline Lung & Breast cancer & EV miR-122 & $\begin{array}{l}\text { Reprograms glucose } \\
\text { metabolism }\end{array}$ & Fong et al., 2015 \\
\hline Lung & Breast cancer & miR-105-rich EVs & Vascular permeability & Zhou et al., 2014 \\
\hline Lung & Breast Cancer & Exosome-associated Annexin II & Angiogenesis & Maji et al., 2017 \\
\hline Lung & Melanoma & $\begin{array}{l}\text { Pigment epithelium-derived factor-positive } \\
\text { exosomes }\end{array}$ & Immune cells recruitment & Plebanek et al., 2017 \\
\hline Lung & Melanoma & Melanoma-derived exosomes & $\begin{array}{l}\text { BMDC recruitment; Vascular } \\
\text { permeability }\end{array}$ & Peinado et al., 2012 \\
\hline Lung & Melanoma & $\begin{array}{l}\text { EVs from insulin-like growth factor } 2 \text { mRNA-binding } \\
\text { protein } 1 \text { (IGF2BP1)-overexpressed/knockdown } \\
\text { melanoma cells }\end{array}$ & $\begin{array}{l}\text { ECM remodeling (fibronectin } \\
\text { deposition); Recruit } \\
\text { CD45 + cells in the lung }\end{array}$ & Ghoshal et al., 2019 \\
\hline Lung & Hepatocellular carcinoma & $\begin{array}{l}\text { Nidogen } 1 \text { (NID1) in metastatic HCC cell-derived } \\
\text { EVs }\end{array}$ & Angiogenesis & Mao et al., 2020 \\
\hline Lung & $\begin{array}{l}\text { High-metastatic } \\
\text { hepatocellular carcinoma }\end{array}$ & miR-1247-3p positive exosomes & Inflammatory microenvironment & Fang et al., 2018 \\
\hline Lung & Colorectal cancer & Exosomal miR-25-3p & $\begin{array}{l}\text { Vascular permeability; } \\
\text { Angiogenesis }\end{array}$ & Zeng et al., 2018 \\
\hline Lung & Colorectal cancer & Integrin beta-like 1-rich-EVs & Inflammatory microenvironment & Ji et al., 2020 \\
\hline Lung & $\begin{array}{l}\text { Pancreatic ductal } \\
\text { adenocarcinomas }\end{array}$ & Exosomal Podocalyxin & ECM remodeling & Novo et al., 2018 \\
\hline Lung & Osteosarcoma & $\begin{array}{l}\text { Osteosarcoma (143-B) cells-derived } \\
\text { EVs-associated TGF } \beta 1\end{array}$ & Fibroblast activation & Mazumdar et al., 2020b \\
\hline Lung & Osteosarcoma & $\begin{array}{l}\text { Highly metastatic 143-B osteosarcoma cell-derived } \\
\text { EVs }\end{array}$ & $\begin{array}{l}\text { CD11b + myeloid cells } \\
\text { recruitment }\end{array}$ & Mazumdar et al., 2020a \\
\hline Lung & Prostate cancer & $\begin{array}{l}\text { Exosomes from human prostate cancer (PCa) PC3 } \\
\text { cells under hypoxic conditions }\end{array}$ & $\begin{array}{l}\text { ECM remodeling; BMDC } \\
\text { recruitment }\end{array}$ & Deep et al., 2020 \\
\hline Lung & Nasopharyngeal carcinoma & EV packaged latent membrane protein 1 & Fibroblast activation & Wu et al., 2020 \\
\hline Lung & SACC & Epiregulin-positive exosomes & $\begin{array}{l}\text { Vascular permeability; } \\
\text { Macrophage recruitment }\end{array}$ & Yang et al., 2017 \\
\hline Lung & Human renal cell carcinoma & MVs derived from CD105-positive cancer stem cells & Angiogenesis & Grange et al., 2011 \\
\hline Lung & $\begin{array}{l}\text { Non-small cell lung } \\
\text { carcinoma (NSCLC) }\end{array}$ & Exosomal RNAs (small nuclear RNAs snRNAs) & Immunosuppression & Liu et al., 2016 \\
\hline Lung & $\begin{array}{l}\text { Metastatic rat } \\
\text { adenocarcinoma }\end{array}$ & $\begin{array}{l}\text { Exosomes from the metastatic rat adenocarcinoma } \\
\text { BSp73ASML (ASML) }\end{array}$ & $\begin{array}{l}\text { ECM remodeling; } \\
\text { Immunosuppression }\end{array}$ & Rana et al., 2013 \\
\hline Liver & Breast Cancer & $\begin{array}{l}\text { Breast cancer derived-EVs-associated nucleoside } \\
\text { diphosphate kinase } A \text { and B(NDPK) }\end{array}$ & Vascular permeability & Duan et al., 2021 \\
\hline Liver & Pancreatic cancer & $\begin{array}{l}\text { Exosomes from the highly metastatic pancreatic } \\
\text { cancer cell line (Panc02-H7 EXO) }\end{array}$ & BMDC recruitment & Yu et al., 2017 \\
\hline Liver & Pancreatic Cancer & EV-associated TGF- $\beta 1$ & Immunosuppression & Zhao et al., 2019 \\
\hline Liver & $\begin{array}{l}\text { Pancreatic ductal } \\
\text { adenocarcinomas }\end{array}$ & $\begin{array}{l}\text { PDAC-derived exosomes-associated macrophage } \\
\text { migration inhibitory factor (MIF) }\end{array}$ & $\begin{array}{l}\text { ECM remodeling; BMDC } \\
\text { recruitment }\end{array}$ & Costa-Silva et al., 2015 \\
\hline Liver & Gastric cancer & EGFR-containing EVs & ECM remodeling & Zhang et al., 2017 \\
\hline Liver & Colorectal cancer & Exosomal miR-25-3p & $\begin{array}{l}\text { Vascular permeability; } \\
\text { Angiogenesis }\end{array}$ & Zeng et al., 2018 \\
\hline Liver & Colorectal cancer & Integrin beta-like 1-rich-EVs & Inflammatory microenvironment & Ji et al., 2020 \\
\hline Bone & Lung cancer & miR-192-enriched- exosome-like vesicles (ELV) & Angiogenesis & Valencia et al., 2014 \\
\hline Bone & Prostate cancer & $\begin{array}{l}\text { Phospholipase D (PLD) isoforms PLD2-riched } \\
\text { exosomes }\end{array}$ & $\begin{array}{l}\text { Shift the bone balance in favor } \\
\text { of osteoblasts }\end{array}$ & Borel et al., 2020 \\
\hline Bone & Prostate cancer & $\begin{array}{l}\text { Enzalutamide resistant (EnzR) CWR-R1 cells } \\
\text { derived EVs (EnzR EVs) }\end{array}$ & BMDC recruitment & Henrich et al., 2020 \\
\hline Brain & $\begin{array}{l}\text { Breast cancer and } \\
\text { melanoma }\end{array}$ & $\begin{array}{l}\text { EVs derived from brain metastases cancer cells } \\
\text { (Br-EVs) }\end{array}$ & $\begin{array}{l}\text { Blood-brain barrier (BBB) } \\
\text { permeability }\end{array}$ & Busatto et al., 2020 \\
\hline Brain & Glioblastoma multiforme & EV-associated VEGF-A & Angiogenesis & Treps et al., 2017 \\
\hline LNs & $\begin{array}{l}\text { Metastatic rat } \\
\text { adenocarcinoma }\end{array}$ & $\begin{array}{l}\text { Exosomes from the metastatic rat adenocarcinoma } \\
\text { BSp73ASML (ASML) }\end{array}$ & $\begin{array}{l}\text { ECM remodeling; } \\
\text { Immunosuppression }\end{array}$ & Rana et al., 2013 \\
\hline LNs & Melanoma & Melanoma-derived exosomes & ECM remodeling; Angiogenesis & Hood et al., 2011 \\
\hline
\end{tabular}


(ZO-1), occludin, and claudin-5 in endothelial cells, thereby promoting cancer metastasis in the liver and lungs of mice (Zeng et al., 2018). In breast cancer, metastatic cancer cells secrete miR-105-rich exosomes that regulate vascular permeability and promote tumor metastasis by downregulating ZO-1 expression (Zhou et al., 2014). Melanoma-derived exosomes upregulate tumor necrosis factor- $\alpha$ expression in the lung, disrupting endothelial cell-cell junctions and increasing vascular permeability (Peinado et al., 2012). Vascular endothelial growth factor-A (VEGF-A) is carried by EVs derived from glioblastoma stem-like cells, increasing vascular permeability in vivo and the angiogenic potential of human brain endothelial cells (Treps et al., 2017). In addition, nucleoside diphosphate kinase B enriched in EVs derived from triple negative breast cancer cells (MDA-MB-231) enhances pulmonary blood vessel leakage and experimental lung metastasis (Duan et al., 2021).

\section{Extracellular Matrix Remodeling}

Extracellular matrix remodeling, a key event in PMN formation, is characterized by the deposition of new ECM components and the expression of enzymes related to ECM modification. The remodeled ECM provides substrates for incoming cancer cells and increases matrix stiffness, which affects the properties of cancer cells (Erler et al., 2009; Cox et al., 2013; Wu et al., 2021). Several ECM components are involved in PMN formation, including fibronectin (Murgai et al., 2017), tenascinC (Urooj et al., 2020), periostin (Malanchi et al., 2011; Fukuda et al., 2015; Wang et al., 2016), and versican (Kim et al., 2009; Gao et al., 2012). Fibronectin is reported to be upregulated in the livers of mice treated with exosomes from highly metastatic pancreatic cancer cells ( $\mathrm{Yu}$ et al., 2017). Macrophage migration inhibitory factor (MIF) is highly expressed in pancreatic cancer cell-derived exosomes. Uptake of exosomal MIF by liver Kupffer cells causes transforming growth factor- $\beta$ (TGF- $\beta$ ) secretion, leading to the activation of hepatic stellate cells. Fibronectin production by the activated hepatic stellate cells promotes the infiltration of bone marrowderived macrophages and neutrophils in the liver, leading to the formation of the PMN (Costa-Silva et al., 2015). Insulinlike growth factor 2 mRNA-binding protein 1 is rich in melanoma cell EVs and promotes PMN formation in the lungs through the deposition of fibronectin and accumulation of $\mathrm{CD}^{+} 5^{+}$cells (Ghoshal et al., 2019). Exosomes from mutant p53-expressing pancreatic ductal adenocarcinoma cells affect the deposition and remodeling of the ECM by fibroblasts to generate a microenvironment highly supportive of tumor cell migration and invasion (Novo et al., 2018). Proteolytic enzymes play a critical role in ECM remodeling during PMN formation. Matrix metalloproteinases (MMPs) are a family of zinc-dependent endopeptidases that target ECM proteins, and MMP induction is one of the hallmarks of PMN formation. Deep et al. (2020) found that exosomes secreted by prostate cancer cells under hypoxia increase MMP activity and upregulate the expression of MMP-2, MMP-9, fibronectin, and collagen IV in the lung, liver, kidney, and spleen. A growing number of EV-associated miRNA have been involved in the regulation of ECM in regional sites. EV-associated miR-494 and miR-542-3p in metastatic rat adenocarcinoma cells regulate tumor-draining LNs and lung tissue by upregulating MMPs to form the PMN (Rana et al., 2013).

Stromal ECM proteins are mainly produced by fibroblasts. ECM remodeling by activated fibroblasts in the PMN has been reported by several groups. Wu et al. (2020) demonstrated that EVs from nasopharyngeal carcinoma package latent membrane protein 1 and activate the conversion of normal fibroblasts into carcinoma-associated fibroblasts (CAFs), thus, increasing the levels of typical PMN biomarkers, including fibronectin, S100A8, and VEGFR1 in lung and liver tissues. Ji et al. (2020) reported that colorectal cancer cells release integrin beta-like 1 (ITGBL1)-rich EVs that activate fibroblasts through the EVsITGBL1-CAFs-TNFAIP3-NF- $\mathrm{KB}$ axis in the liver and lung; the activated fibroblasts promote PMN formation by secreting pro-inflammatory factors. Fang et al. (2018) found that the highly metastatic hepatocellular carcinoma cell-derived exosomal miR-1247-3p induces the activation of normal fibroblasts into CAFs, which secrete inflammatory cytokines such as IL-6 and IL-8 to promote PMN formation in the lung, thus, promoting lung metastasis of liver cancer. Mazumdar et al. (2020b) provided strong evidence that osteosarcoma cellderived EVs can activate fibroblasts into CAF phenotypes in the lung PMN through EV-associated TGF- $\beta 1$ and SMAD2 pathway activation.

\section{Bone Marrow-Derived Cell Recruitment}

One mechanism by which tumor factors promote PMN formation is by mobilizing BMDCs to establish a suitable environment in specific secondary organs. The protooncoprotein MET is a receptor tyrosine kinase involved in cancer cell growth and invasion. Exosomes from highly metastatic melanomas reprogram BMDCs toward a provasculogenic phenotype by transferring EV-associated MET, and thus, increasing the metastatic behavior of primary tumors (Peinado et al., 2012). Osteosarcoma-derived EVs can increase $\mathrm{CD}_{11 \mathrm{~b}^{+}}$myeloid cell infiltration in the lungs (Mazumdar et al., 2020a). In pancreatic cancer, highly metastatic pancreatic cancer cell-derived exosomes recruit $\mathrm{CD}_{11 \mathrm{~b}}{ }^{+}$and $\mathrm{CD} 45^{+}$ hematopoietic progenitor cells at the PMN (Yu et al., 2017). An opposite example of tumor EVs promoting PMN formation is the inhibition of lung metastasis in melanoma cells with low metastatic potential (Plebanek et al., 2017). These low-metastatic tumor cell-derived exosomes can amplify Ly6C ${ }^{\text {low }}$ patrolling monocytes in the bone marrow and trigger a wide range of innate immune responses. The pigment epithelial-derived factor on the external surface of exosomes plays a critical role in the process.

\section{Angiogenesis}

Angiogenesis is a prominent characteristic of the PMN. It was demonstrated that treating immunodeficient mice with epiregulin-enriched exosomes derived from salivary adenoid cystic carcinoma greatly enhanced tumor metastasis to the lung. Epiregulin-enriched exosomes upregulate the expression of VEGF, FGF-2, IL-8, and VEGFR1 in lung vascular endothelial cells, thus, contributing to angiogenesis (Yang et al., 2017). 
Human renal cancer stem cells promote angiogenesis and the formation of a PMN in the lung; the process involves the induction of pro-angiogenic mRNAs and miRNAs by CD105 microvesicles in the whole organ (Grange et al., 2011). Nidogen 1 in metastatic hepatocellular carcinoma cell-derived EVs is reported to promote PMN formation in the lung by enhancing angiogenesis and pulmonary endothelial permeability to facilitate colonization of tumor cells. EV-associated nidogen 1 activates fibroblasts to secrete tumor necrosis factor receptor 1, thereby facilitating lung colonization of tumor cells (Mao et al., 2020). Annexin II is one of the most highly expressed proteins in exosomes. Maji et al. (2017) showed that exosomal Annexin II generates a PMN to facilitate breast cancer metastasis in distant organs by promoting tissue plasminogen activatordependent angiogenesis.

\section{Immunosuppression}

The PMN is an immunosuppressive microenvironment comprising $\mathrm{T}$ cells, natural killer (NK) cells, neutrophils, monocytes, and macrophages (Seubert et al., 2015; Patel et al., 2018). Wen et al. (2016) reported that exosomes derived from highly metastatic murine breast cancer cells are distributed predominantly to the lungs of mice, where they suppress T-cell proliferation and inhibit NK cell cytotoxicity, likely suppressing the anticancer immune response in premetastatic organs. EVs isolated from pancreatic cancer induce a dysfunctional phenotype in NK cells, which contributes to an immunosuppressive microenvironment in the liver, and ultimately results in PMN formation. The study provided evidence that pancreatic cancer-derived EVs induce Smad2/3 phosphorylation and downregulate NKG2D in NK cells by delivering TGF- $\beta 1$ to $\mathrm{NK}$ cells, which contributes to PMN formation in liver (Zhao et al., 2019). It is demonstrated that lung alveolar epithelial cells are stimulated by tumor exosomal RNAs via Toll-like receptor 3, which triggers neutrophil recruitment and lung metastatic niche formation (Liu et al., 2016). EVs secreted by brain metastases cells cause low-density lipoprotein aggregation, which accelerates EVs uptake by monocytes and macrophages. These monocytes and macrophages secrete immunosuppressive factors such as interleukin 10, chemokine ligand 2, and TGF- $\beta$, which contribute to PMN formation (Busatto et al., 2020). Maus et al. (2019) investigated lymphatic EVs of melanoma patients and showed that EVs traffic from the primary tumor microenvironment to the sentinel LNs and regulate the immune microenvironment in LNs, thereby promoting PMN formation.

\section{Others}

Altered glucose metabolism, a hallmark of cancer, is characterized by increased glycolysis and glucose uptake. Fong et al. (2015) demonstrated that EV-associated microRNA-122 secreted by breast cancer cells can be transferred to normal cells (lung fibroblasts, brain astrocytes, and neurons) in the PMN, leading to reduced glucose uptake in these cells. Thereby, the niche accommodates a massive energy for cancer cell metastatic growth by suppressing the nutrient utilization in other cell types.

\section{PRE-METASTATIC NICHE FORMATION IN DIFFERENT ORGANS}

\section{Lung}

Our understanding of PMN biology is mostly based on studies of lung metastasis. Tumor EVs can target lung endothelial cells and cause vascular leakage and angiogenesis at premetastatic sites (Grange et al., 2011; Peinado et al., 2012; Zhou et al., 2014; Maji et al., 2017; Yang et al., 2017; Zeng et al., 2018; Mao et al., 2020). Lung fibroblasts are another common target of tumor EVs at PMN sites. Tumor EVs induce lung fibroblast reprogramming, by which fibroblasts are activated and differentiate into myofibroblast/CAF phenotypes, resulting in ECM remodeling, angiogenesis, secretion of pro-inflammatory cytokines, and immunosuppression (Fang et al., 2018; Novo et al., 2018; Ji et al., 2020; Mazumdar et al., 2020b; Wu et al., 2020). Alveolar epithelial cells released from tumor EVs secrete chemokines and neutrophils in the lungs to promote the formation of the microenvironment before lung metastasis (Liu et al., 2016). In addition, BMDCs $\left(\mathrm{CD} 45^{+}\right)$from tumor exosomes in the lung inhibit the proliferation of $\mathrm{T}$ cells and the cytotoxicity of NK cells to form an immunosuppressive microenvironment (Wen et al., 2016). Overall, EVs target lung endothelial cells, fibroblasts, and alveolar epithelial cells to construct an inflammatory and immunosuppressive niche for the colonization of circulating tumor cells.

\section{Liver}

Tumor EVs can be internalized by Kupffer cells (F4/80 positive) and hepatic stellate cells (alpha smooth muscle actin and desmin positive) in the liver and activate downstream signaling pathways. Costa-Silva et al. showed that macrophage MIF is highly expressed in pancreatic cancer-derived exosomes. Uptake of these exosomes by liver Kupffer cells causes TGF- $\beta$ secretion, leading to activation of hepatic stellate cells. Fibronectin production by activated hepatic stellate cells promotes the infiltration of bone marrow-derived macrophages and neutrophils in the liver, leading to the formation of the PMN (Costa-Silva et al., 2015). In gastric cancer cells, epidermal growth factor receptor-containing exosomes target Kupffer cells and hepatic stellate cells to favor the development of a liver-like microenvironment, thereby promoting liver-specific metastasis (Zhang et al., 2017). In addition, cancer-derived EVs may target NK cells in the liver. Zhao et al. (2019) demonstrated that pancreatic cancer-derived EVs induce a dysfunctional phenotype of NK cells, which contributes to an immunosuppressive microenvironment in the liver, and ultimately results in PMN formation. These studies indicate that EVs establish a fibrotic and immunosuppressive liver PMN mainly through Kupffer cells and hepatic stellate cells, but not hepatocytes. It suggests that targeting circulation EVs and/or the fibrotic niche may provide an early prevention and therapeutic intervention for liver metastasis.

\section{Brain}

Disruption of the blood-brain barrier is related to metastatic initiation and progression. Treps et al. (2017) reported 
that VEGF-A is carried by EVs derived from glioblastoma stemlike cells, and VEGF-A-enriched EVs promote PMN formation in the brain by targeting endothelial cells and increasing vascular permeability. In the same study, they demonstrated that EV-associated VEGF-A exerts pro-angiogenic activity on brain endothelial cells to stimulate angiogenesis. In addition, EVs may target non-endothelial cells in the brain to promote cancer brain metastasis. Busatto et al. revealed that brain metastasis cellderived EVs interact with low-density lipoprotein and accelerate EV uptake by monocytes. These monocytes are key components in the brain niche and secrete immunosuppressive factors, such as interleukin 10, chemokine ligand 2 , and TGF- $\beta$, which contribute to PMN formation (Busatto et al., 2020).

\section{Bone Marrow}

Bone marrow myeloid cells and osteoblasts may contribute to PMN formation in the bone marrow. Henrich et al. characterized EV-mediated communication between prostate cancer cells and bone marrow myeloid cells, and demonstrated that cholesterol homeostasis in bone marrow myeloid cells regulates prometastatic EV signaling and metastasis. The phospholipase $\mathrm{D}$ (PLD) isoforms PLD1/2 regulate tumor progression and metastasis by catalyzing the hydrolysis of phosphatidylcholine to yield phosphatidic acid (Henrich et al., 2020). Borel et al. (2020) demonstrated that PLD2 is present in EVs of prostate cancer cells and activates proliferation and differentiation of osteoblasts by stimulating ERK $1 / 2$ phosphorylation, leading to the formation of a microenvironment before bone metastasis. By contrast, Valencia et al. reported that lung cancer cells release miR-192-enriched EVs, which target endothelial cells and show antimetastatic activity in vivo. EV-associated miR-192 is internalized by endothelial cells and inhibits the expression of proangiogenic factors including IL-8, ICAM, and CXCL1 to reduce metastatic colonization (Valencia et al., 2014). Bone marrow is a complex microenvironment and contains multiple cell types such as osteoblasts, osteoclasts, myeloid cells, fibroblasts, macrophages, adipocytes, and endothelial cells. More studies are required to elucidate how these cells cooperate in $\mathrm{PMN}$ formation in the bone marrow.

\section{Lymph Nodes}

Metastasis to LNs is common in various cancers. Melanomaderived exosomes home to sentinel LNs and can convert a remote $\mathrm{LN}$ into a PMN before tumor cell colonization by inducing the expression of factors responsible for cell recruitment, matrix remodeling, and angiogenesis (Hood et al., 2011). Maus et al. (2019) investigated lymphatic EVs of melanoma patients and showed that EVs traffic from the primary tumor microenvironment to the sentinel LNs, where they regulate the

\section{REFERENCES}

Aguado, B. A., Caffe, J. R., Nanavati, D., Rao, S. S., Bushnell, G. G., Azarin, S. M., et al. (2016). Extracellular matrix mediators of metastatic cell colonization characterized using scaffold mimics of the pre-metastatic niche. Acta Biomater. 33, 13-24. doi: 10.1016/j.actbio.2016.01.043 immune microenvironment, thereby initiating PMN formation. However, these studies did not identify the definite target cells of tumor EVs in LNs.

\section{CLINICAL IMPLICATIONS AND FUTURE PERSPECTIVES}

Studies show that various cancers can promote PMN formation in the lung, liver, brain, bone, and LNs by targeting endothelial cells, fibroblasts, alveolar epithelial cells, Kupffer cells, hepatic stellate cells, monocytes, and macrophages in these organs. Tumor EVs play a critical role in the communication between the primary tumor and distant organs by precisely delivering tumor products to target cells. Thus, tumor EVs in circulation should be a potential target of liquid biopsy to predict metastasis. Blocking the delivery of tumor EVs to target cells may prevent cancer metastasis. However, the biomarkers of tumor EVs that promote PMN formation have not been fully identified, and the mechanisms underlying the uptake of tumor EVs by target cells remain unclear. Except tumor cell-derived EVs, our group demonstrated that EVs secreted by CAFs can promote PMN formation in the lung by activating lung fibroblasts (Kong et al., 2019). Further study is necessary to determine whether EVs from other stromal cells in the primary tumor can induce PMN formation in more distant organs. Potential future studies in the field may focus on the following: (1) the different roles of EVs secreted by tumor and stromal cells in PMN formation; (2) the biomarkers of tumor or stromal EVs that promote PMN formation in various cancers; (3) the mechanisms by which recipient cells take up tumor or stromal EVs. The identification of PMN characteristics and a better understanding of the roles of EVs in PMN formation in various organs may help prevent cancer metastasis at an early stage.

\section{AUTHOR CONTRIBUTIONS}

QD, XL, KC, JS, and JK collected the literatures. TL and QD prepared the manuscript. All authors contributed to the article and approved the submitted version.

\section{FUNDING}

This work was supported by National Natural Science Foundation of China (82073001), LiaoNing Revitalization Talent Program (XLYC1805006), and Dalian Science and Technology Innovation Project (2020JJ26SN053). 
amplified oncogene sequences. Nat. Commun. 2:180. doi: 10.1038/ncomms 1180

Borel, M., Lollo, G., Magne, D., Buchet, R., Brizuela, L., and Mebarek, S. (2020). Prostate cancer-derived exosomes promote osteoblast differentiation and activity through phospholipase D2. Biochim. Biophys. Acta Mol. Basis Dis. 1866:165919. doi: 10.1016/j.bbadis.2020.165919

Busatto, S., Yang, Y., Walker, S. A., Davidovich, I., Lin, W. H., Lewis-Tuffin, L., et al. (2020). Brain metastases-derived extracellular vesicles induce binding and aggregation of low-density lipoprotein. J. Nanobiotechnol. 18:162. doi: 10.1186/ s12951-020-00722-2

Chen, Q., Zhang, X. H., and Massague, J. (2011). Macrophage binding to receptor VCAM-1 transmits survival signals in breast cancer cells that invade the lungs. Cancer Cell 20, 538-549. doi: 10.1016/j.ccr.2011.08.025

Choi, D. S., Kim, D. K., Kim, Y. K., and Gho, Y. S. (2013). Proteomics, transcriptomics and lipidomics of exosomes and ectosomes. Proteomics 13, 1554-1571. doi: 10.1002/pmic.201200329

Colombo, M., Raposo, G., and Thery, C. (2014). Biogenesis, secretion, and intercellular interactions of exosomes and other extracellular vesicles. Annu. Rev. Cell Dev. Biol. 30, 255-289. doi: 10.1146/annurev-cellbio-101512-122326

Costa-Silva, B., Aiello, N. M., Ocean, A. J., Singh, S., Zhang, H., Thakur, B. K., et al. (2015). Pancreatic cancer exosomes initiate pre-metastatic niche formation in the liver. Nat. Cell Biol. 17, 816-826. doi: 10.1038/ncb3169

Cox, T. R., Bird, D., Baker, A. M., Barker, H. E., Ho, M. W., Lang, G., et al. (2013). LOX-mediated collagen crosslinking is responsible for fibrosisenhanced metastasis. Cancer Res. 73, 1721-1732. doi: 10.1158/0008-5472.CAN12-2233

Deep, G., Jain, A., Kumar, A., Agarwal, C., Kim, S., Leevy, W. M., et al. (2020). Exosomes secreted by prostate cancer cells under hypoxia promote matrix metalloproteinases activity at pre-metastatic niches. Mol. Carcinog. 59, 323332. doi: $10.1002 / \mathrm{mc} .23157$

Duan, S., Nordmeier, S., Byrnes, A. E., and Buxton, I. L. O. (2021). Extracellular Vesicle-Mediated Purinergic Signaling Contributes to Host Microenvironment Plasticity and Metastasis in Triple Negative Breast Cancer. Int. J. Mol. Sci. 22:597. doi: 10.3390/ijms22020597

Erler, J. T., Bennewith, K. L., Cox, T. R., Lang, G., Bird, D., Koong, A., et al. (2009). Hypoxia-induced lysyl oxidase is a critical mediator of bone marrow cell recruitment to form the premetastatic niche. Cancer Cell 15, 35-44. doi: 10.1016/j.ccr.2008.11.012

Fang, T., Lv, H., Lv, G., Li, T., Wang, C., Han, Q., et al. (2018). Tumor-derived exosomal miR-1247-3p induces cancer-associated fibroblast activation to foster lung metastasis of liver cancer. Nat. Commun. 9:191. doi: 10.1038/s41467-01702583-0

Fong, M. Y., Zhou, W., Liu, L., Alontaga, A. Y., Chandra, M., Ashby, J., et al. (2015). Breast-cancer-secreted miR-122 reprograms glucose metabolism in premetastatic niche to promote metastasis. Nat. Cell Biol. 17, 183-194. doi: $10.1038 /$ ncb3094

Fukuda, K., Sugihara, E., Ohta, S., Izuhara, K., Funakoshi, T., Amagai, M., et al. (2015). Periostin Is a Key Niche Component for Wound Metastasis of Melanoma. PLoS One 10:e0129704. doi: 10.1371/journal.pone.0129704

Gao, D., Joshi, N., Choi, H., Ryu, S., Hahn, M., Catena, R., et al. (2012). Myeloid progenitor cells in the premetastatic lung promote metastases by inducing mesenchymal to epithelial transition. Cancer Res. 72, 1384-1394. doi: 10.1158/ 0008-5472.CAN-11-2905

Ghoshal, A., Rodrigues, L. C., Gowda, C. P., Elcheva, I. A., Liu, Z., Abraham, T., et al. (2019). Extracellular vesicle-dependent effect of RNA-binding protein IGF2BP1 on melanoma metastasis. Oncogene 38, 4182-4196. doi: 10.1038/ s41388-019-0797-3

Giles, A. J., Reid, C. M., Evans, J. D., Murgai, M., Vicioso, Y., Highfill, S. L., et al. (2016). Activation of Hematopoietic Stem/Progenitor Cells Promotes Immunosuppression Within the Pre-metastatic Niche. Cancer Res. 76, 13351347. doi: 10.1158/0008-5472.CAN-15-0204

Grange, C., Tapparo, M., Collino, F., Vitillo, L., Damasco, C., Deregibus, M. C., et al. (2011). Microvesicles released from human renal cancer stem cells stimulate angiogenesis and formation of lung premetastatic niche. Cancer Res. 71, 53465356. doi: 10.1158/0008-5472.CAN-11-0241

Gupta, G. P., Nguyen, D. X., Chiang, A. C., Bos, P. D., Kim, J. Y., Nadal, C., et al. (2007). Mediators of vascular remodelling co-opted for sequential steps in lung metastasis. Nature 446, 765-770. doi: 10.1038/nature05760
Henrich, S. E., McMahon, K. M., Plebanek, M. P., Calvert, A. E., Feliciano, T. J., Parrish, S., et al. (2020). Prostate cancer extracellular vesicles mediate intercellular communication with bone marrow cells and promote metastasis in a cholesterol-dependent manner. J. Extracell. Vesicles 10:e12042. doi: 10.1002/ jev2.12042

Hiratsuka, S., Nakamura, K., Iwai, S., Murakami, M., Itoh, T., Kijima, H., et al. (2002). MMP9 induction by vascular endothelial growth factor receptor-1 is involved in lung-specific metastasis. Cancer Cell 2, 289-300. doi: 10.1016/ s1535-6108(02)00153-8

Hood, J. L., San, R. S., and Wickline, S. A. (2011). Exosomes released by melanoma cells prepare sentinel lymph nodes for tumor metastasis. Cancer Res. 71, 3792-3801. doi: 10.1158/0008-5472.CAN-10-4455

Hoshino, A., Costa-Silva, B., Shen, T. L., Rodrigues, G., Hashimoto, A., Tesic Mark, M., et al. (2015). Tumour exosome integrins determine organotropic metastasis. Nature 527, 329-335. doi: 10.1038/nature15756

Houg, D. S., and Bijlsma, M. F. (2018). The hepatic pre-metastatic niche in pancreatic ductal adenocarcinoma. Mol. Cancer 17:95. doi: 10.1186/s12943018-0842-9

Huang, Y., Song, N., Ding, Y., Yuan, S., Li, X., Cai, H., et al. (2009). Pulmonary vascular destabilization in the premetastatic phase facilitates lung metastasis. Cancer Res. 69, 7529-7537. doi: 10.1158/0008-5472.CAN-08-4382

Ji, Q., Zhou, L., Sui, H., Yang, L., Wu, X., Song, Q., et al. (2020). Primary tumors release ITGBL1-rich extracellular vesicles to promote distal metastatic tumor growth through fibroblast-niche formation. Nat. Commun. 11:1211. doi: 10. 1038/s41467-020-14869-x

Kaplan, R. N., Psaila, B., and Lyden, D. (2007). Niche-to-niche migration of bonemarrow-derived cells. Trends Mol. Med. 13, 72-81. doi: 10.1016/j.molmed.2006. 12.003

Kaplan, R. N., Riba, R. D., Zacharoulis, S., Bramley, A. H., Vincent, L., Costa, C., et al. (2005). VEGFR1-positive haematopoietic bone marrow progenitors initiate the pre-metastatic niche. Nature 438, 820-827. doi: 10.1038/ nature 04186

Kim, H., Chung, H., Kim, J., Choi, D. H., Shin, Y., Kang, Y. G., et al. (2019). Macrophages-Triggered Sequential Remodeling of EndotheliumInterstitial Matrix to Form Pre-Metastatic Niche in Microfluidic Tumor Microenvironment. Adv. Sci. 6:1900195. doi: 10.1002/advs.20190 0195

Kim, S., Takahashi, H., Lin, W. W., Descargues, P., Grivennikov, S., Kim, Y., et al. (2009). Carcinoma-produced factors activate myeloid cells through TLR2 to stimulate metastasis. Nature 457, 102-106. doi: 10.1038/nature07623

Kitamura, T., Qian, B. Z., and Pollard, J. W. (2015). Immune cell promotion of metastasis. Nat. Rev. Immunol. 15, 73-86. doi: 10.1038/nri3789

Kong, J., Tian, H., Zhang, F., Zhang, Z., Li, J., Liu, X., et al. (2019). Extracellular vesicles of carcinoma-associated fibroblasts creates a pre-metastatic niche in the lung through activating fibroblasts. Mol. Cancer 18:175. doi: 10.1186/s12943019-1101-4

Liu, Y., Gu, Y., Han, Y., Zhang, Q., Jiang, Z., Zhang, X., et al. (2016). Tumor Exosomal RNAs Promote Lung Pre-metastatic Niche Formation by Activating Alveolar Epithelial TLR3 to Recruit Neutrophils. Cancer Cell. 30, 243-256. doi: 10.1016/j.ccell.2016.06.021

Maji, S., Chaudhary, P., Akopova, I., Nguyen, P. M., Hare, R. J., Gryczynski, I., et al. (2017). Exosomal Annexin II Promotes Angiogenesis and Breast Cancer Metastasis. Mol. Cancer Res. 15, 93-105. doi: 10.1158/1541-7786.MCR-160163

Malanchi, I., Santamaria-Martinez, A., Susanto, E., Peng, H., Lehr, H. A., Delaloye, J. F., et al. (2011). Interactions between cancer stem cells and their niche govern metastatic colonization. Nature 481, 85-89. doi: 10.1038/nature10694

Mao, X., Tey, S. K., Yeung, C. L. S., Kwong, E. M. L., Fung, Y. M. E., Chung, C. Y. S., et al. (2020). Nidogen 1-Enriched Extracellular Vesicles Facilitate Extrahepatic Metastasis of Liver Cancer by Activating Pulmonary Fibroblasts to Secrete Tumor Necrosis Factor Receptor 1. Adv. Sci. 7:2002157. doi: 10.1002/ advs.202002157

Maus, R. L. G., Jakub, J. W., Hieken, T. J., Nevala, W. K., Christensen, T. A., Sutor, S. L., et al. (2019). Identification of novel, immune-mediating extracellular vesicles in human lymphatic effluent draining primary cutaneous melanoma. Oncoimmunology 8:e1667742. doi: 10.1080/2162402X.2019.1667742

Mazumdar, A., Urdinez, J., Boro, A., Arlt, M. J. E., Egli, F. E., Niederost, B., et al. (2020a). Exploring the Role of Osteosarcoma-Derived Extracellular Vesicles in 
Pre-Metastatic Niche Formation and Metastasis in the 143-B Xenograft Mouse Osteosarcoma Model. Cancers 12:3457. doi: 10.3390/cancers12113457

Mazumdar, A., Urdinez, J., Boro, A., Migliavacca, J., Arlt, M. J. E., Muff, R., et al. (2020b). Osteosarcoma-Derived Extracellular Vesicles Induce Lung Fibroblast Reprogramming. Int. J. Mol. Sci. 21:5451. doi: 10.3390/ijms21155451

Mohan, V., Das, A., and Sagi, I. (2020). Emerging roles of ECM remodeling processes in cancer. Semin. Cancer Biol. 62, 192-200. doi: 10.1016/j.semcancer. 2019.09.004

Murgai, M., Ju, W., Eason, M., Kline, J., Beury, D. W., Kaczanowska, S., et al. (2017). KLF4-dependent perivascular cell plasticity mediates pre-metastatic niche formation and metastasis. Nat. Med. 23, 1176-1190. doi: 10.1038/nm. 4400

Novo, D., Heath, N., Mitchell, L., Caligiuri, G., MacFarlane, A., Reijmer, D., et al. (2018). Mutant p53s generate pro-invasive niches by influencing exosome podocalyxin levels. Nat. Commun. 9:5069. doi: 10.1038/s41467-018-07339-y

Patel, S., Fu, S., Mastio, J., Dominguez, G. A., Purohit, A., Kossenkov, A., et al. (2018). Unique pattern of neutrophil migration and function during tumor progression. Nat. Immunol. 19, 1236-1247. doi: 10.1038/s41590-018-0229-5

Peinado, H., Aleckovic, M., Lavotshkin, S., Matei, I., Costa-Silva, B., MorenoBueno, G., et al. (2012). Melanoma exosomes educate bone marrow progenitor cells toward a pro-metastatic phenotype through MET. Nat. Med. 18, 883-891. doi: $10.1038 / \mathrm{nm} .2753$

Plebanek, M. P., Angeloni, N. L., Vinokour, E., Li, J., Henkin, A., Martinez-Marin, D., et al. (2017). Pre-metastatic cancer exosomes induce immune surveillance by patrolling monocytes at the metastatic niche. Nat. Commun. 8:1319. doi: 10.1038/s41467-017-01433-3

Rana, S., Malinowska, K., and Zoller, M. (2013). Exosomal tumor microRNA modulates premetastatic organ cells. Neoplasia 15, 281-295. doi: 10.1593/neo. 122010

Raposo, G., and Stoorvogel, W. (2013). Extracellular vesicles: exosomes, microvesicles, and friends. J. Cell Biol. 200, 373-383. doi: 10.1083/jcb. 201211138

Seubert, B., Grunwald, B., Kobuch, J., Cui, H., Schelter, F., Schaten, S., et al. (2015). Tissue inhibitor of metalloproteinases (TIMP)-1 creates a premetastatic niche in the liver through SDF-1/CXCR4-dependent neutrophil recruitment in mice. Hepatology 61, 238-248. doi: 10.1002/hep.27378

Sun, H., Meng, Q., Shi, C., Yang, H., Li, X., Wu, S., et al. (2021). Hypoxiainducible exosomes facilitate liver-tropic pre-metastatic niche in colorectal cancer. Hepatology doi: 10.1002/hep.32009 [Epub ahead of print].

Tacke, R. S., Lee, H. C., Goh, C., Courtney, J., Polyak, S. J., Rosen, H. R., et al. (2012). Myeloid suppressor cells induced by hepatitis C virus suppress T-cell responses through the production of reactive oxygen species. Hepatology 55, 343-353. doi: 10.1002/hep. 24700

Thakur, B. K., Zhang, H., Becker, A., Matei, I., Huang, Y., Costa-Silva, B., et al. (2014). Double-stranded DNA in exosomes: a novel biomarker in cancer detection. Cell Res. 24, 766-769. doi: 10.1038/cr.2014.44

Thery, C., Witwer, K. W., Aikawa, E., Alcaraz, M. J., Anderson, J. D., Andriantsitohaina, R., et al. (2018). Minimal information for studies of extracellular vesicles 2018 (MISEV2018): a position statement of the International Society for Extracellular Vesicles and update of the MISEV2014 guidelines. J. Extracell. Vesicles 7:1535750. doi: 10.1080/20013078.2018. 1535750

Treps, L., Perret, R., Edmond, S., Ricard, D., and Gavard, J. (2017). Glioblastoma stem-like cells secrete the pro-angiogenic VEGF-A factor in extracellular vesicles. J. Extracell. Vesicles 6:1359479. doi: 10.1080/20013078.2017.135 9479

Tyagi, A., Sharma, S., Wu, K., Wu, S. Y., Xing, F., Liu, Y., et al. (2021). Nicotine promotes breast cancer metastasis by stimulating N2 neutrophils and generating pre-metastatic niche in lung. Nat. Commun. 12:474. doi: 10.1038/ s41467-020-20733-9

Urooj, T., Wasim, B., Mushtaq, S., Shah, S. N. N., and Shah, M. (2020). Cancer Cell-derived Secretory Factors in Breast Cancer-associated Lung Metastasis: their Mechanism and Future Prospects. Curr. Cancer Drug Targets 20, 168-186. doi: 10.2174/1568009620666191220151856

Valadi, H., Ekstrom, K., Bossios, A., Sjostrand, M., Lee, J. J., and Lotvall, J. O. (2007). Exosome-mediated transfer of mRNAs and microRNAs is a novel mechanism of genetic exchange between cells. Nat. Cell Biol. 9, 654-659. doi: 10.1038/ncb1596

Valencia, K., Luis-Ravelo, D., Bovy, N., Anton, I., Martinez-Canarias, S., Zandueta, C., et al. (2014). miRNA cargo within exosome-like vesicle transfer influences metastatic bone colonization. Mol. Oncol. 8, 689-703. doi: 10.1016/j.molonc. 2014.01.012

van Niel, G., D'Angelo, G., and Raposo, G. (2018). Shedding light on the cell biology of extracellular vesicles. Nat. Rev. Mol. Cell Biol. 19, 213-228. doi: 10.1038/nrm.2017.125

Wang, Y., Ding, Y., Guo, N., and Wang, S. (2019). MDSCs: key Criminals of Tumor Pre-metastatic Niche Formation. Front. Immunol. 10:172. doi: 10.3389/fimmu. 2019.00172

Wang, Z., Xiong, S., Mao, Y., Chen, M., Ma, X., Zhou, X., et al. (2016). Periostin promotes immunosuppressive premetastatic niche formation to facilitate breast tumour metastasis. J. Pathol. 239, 484-495. doi: 10.1002/path.4747

Wen, S. W., Sceneay, J., Lima, L. G., Wong, C. S., Becker, M., Krumeich, S., et al. (2016). The Biodistribution and Immune Suppressive Effects of Breast CancerDerived Exosomes. Cancer Res. 76, 6816-6827. doi: 10.1158/0008-5472.CAN16-0868

Wu, S., Xing, X., Wang, Y., Zhang, X., Li, M., Wang, M., et al. (2021). The pathological significance of LOXL2 in pre-metastatic niche formation of HCC and its related molecular mechanism. Eur. J. Cancer 147, 63-73. doi: 10.1016/j. ejca.2021.01.011

Wu, X., Zhou, Z., Xu, S., Liao, C., Chen, X., Li, B., et al. (2020). Extracellular vesicle packaged LMP1-activated fibroblasts promote tumor progression via autophagy and stroma-tumor metabolism coupling. Cancer Lett. 478, 93-106. doi: 10.1016/j.canlet.2020.03.004

Xu, W. W., Li, B., Guan, X. Y., Chung, S. K., Wang, Y., Yip, Y. L., et al. (2017). Cancer cell-secreted IGF2 instigates fibroblasts and bone marrowderived vascular progenitor cells to promote cancer progression. Nat. Commun. 8:14399. doi: 10.1038/ncomms14399

Yang, W. W., Yang, L. Q., Zhao, F., Chen, C. W., Xu, L. H., Fu, J., et al. (2017). Epiregulin Promotes Lung Metastasis of Salivary Adenoid Cystic Carcinoma. Theranostics 7, 3700-3714. doi: 10.7150/thno.19712

You, H., Baluszek, S., and Kaminska, B. (2020). Supportive roles of brain macrophages in CNS metastases and assessment of new approaches targeting their functions. Theranostics 10, 2949-2964. doi: 10.7150/thno.40783

Yu, Z., Zhao, S., Ren, L., Wang, L., Chen, Z., Hoffman, R. M., et al. (2017). Pancreatic cancer-derived exosomes promote tumor metastasis and liver pre-metastatic niche formation. Oncotarget 8, 63461-63483. doi: 10.18632/ oncotarget.18831

Zeng, Z., Li, Y., Pan, Y., Lan, X., Song, F., Sun, J., et al. (2018). Cancer-derived exosomal miR-25-3p promotes pre-metastatic niche formation by inducing vascular permeability and angiogenesis. Nat. Commun. 9:5395. doi: 10.1038/ s41467-018-07810-w

Zhang, H., Deng, T., Liu, R., Bai, M., Zhou, L., Wang, X., et al. (2017). Exosomedelivered EGFR regulates liver microenvironment to promote gastric cancer liver metastasis. Nat. Commun. 8:15016. doi: 10.1038/ncomms15016

Zhang, Y., and Wang, X. F. (2015). A niche role for cancer exosomes in metastasis. Nat. Cell Biol. 17, 709-711. doi: 10.1038/ncb3181

Zhao, J., Schlosser, H. A., Wang, Z., Qin, J., Li, J., Popp, F., et al. (2019). TumorDerived Extracellular Vesicles Inhibit Natural Killer Cell Function in Pancreatic Cancer. Cancers 11:874. doi: 10.3390/cancers11060874

Zhou, W., Fong, M. Y., Min, Y., Somlo, G., Liu, L., Palomares, M. R., et al. (2014). Cancer-secreted miR-105 destroys vascular endothelial barriers to promote metastasis. Cancer Cell 25, 501-515. doi: 10.1016/j.ccr.2014.03.007

Conflict of Interest: The authors declare that the research was conducted in the absence of any commercial or financial relationships that could be construed as a potential conflict of interest.

Publisher's Note: All claims expressed in this article are solely those of the authors and do not necessarily represent those of their affiliated organizations, or those of the publisher, the editors and the reviewers. Any product that may be evaluated in this article, or claim that may be made by its manufacturer, is not guaranteed or endorsed by the publisher.

Copyright (c) 2021 Dong, Liu, Cheng, Sheng, Kong and Liu. This is an open-access article distributed under the terms of the Creative Commons Attribution License (CC BY). The use, distribution or reproduction in other forums is permitted, provided the original author(s) and the copyright owner(s) are credited and that the original publication in this journal is cited, in accordance with accepted academic practice. No use, distribution or reproduction is permitted which does not comply with these terms. 